\title{
Menopausal status and adjuvant hormonal therapy for breast cancer patients: A practical guideline
}

\author{
F.Y.F.L. De $\operatorname{Vos}^{\text {a }}{ }^{*}$, H.W.M. van Laarhoven ${ }^{\text {b }}{ }^{\text {, J.S.E. Laven }}{ }^{\text {c }}$, A.P.N. Themmen ${ }^{\text {d, }}$ \\ L.V.A.M. Beex ${ }^{\text {e }}$ C.G.J. Sweep ${ }^{\text {f }}$, C. Seynaeve ${ }^{g}$, A. Jager ${ }^{\mathrm{g}}$ \\ ${ }^{a}$ Department of Medical Oncology, University Medical Center Utrecht, Heidelberglaan 100, 3584 CX Utrecht, The Netherlands \\ ${ }^{\mathrm{b}}$ Department of Medical Oncology, Academic Medical Centre, University of Amsterdam, Amsterdam, The Netherlands \\ ${ }^{c}$ Division of Reproductive Medicine, Department of Obstetrics \& Gynaecology, Erasmus University Medical Center, 's-Gravendijkwal 230,3015 CE \\ Rotterdam, The Netherlands \\ ${ }^{\mathrm{d}}$ Department of Internal Medicine, Erasmus University Medical Center, 's-Gravendijkwal 230, 3015 CE Rotterdam, The Netherlands \\ ${ }^{\mathrm{e}}$ Department of Medical Oncology, Radboud University Nijmegen Medical Center, Geert Grooteplein-zuid 10, 6525 GA Nijmegen, The Netherlands \\ ${ }^{\mathrm{f}}$ Department of Laboratory Medicine, Radboud University Nijmegen Medical Center, Geert Grooteplein-zuid 10, 6525 GA Nijmegen, The Netherlands \\ g Department of Medical Oncology, Erasmus University Medical Center-Daniel den Hoed Cancer Center, Groene Hilledijk 301, 3075 EA Rotterdam, The \\ Netherlands
}

Accepted 20 June 2012

\section{Contents}

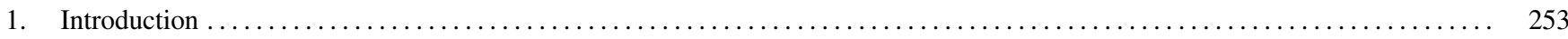

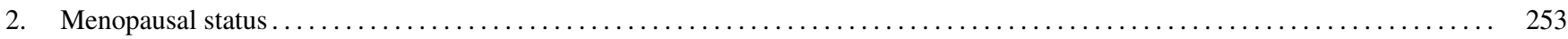

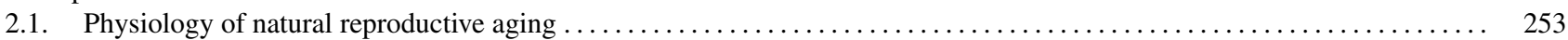

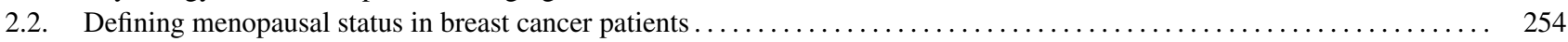

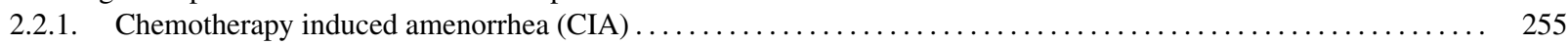

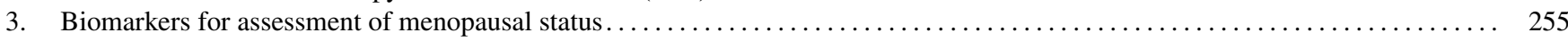

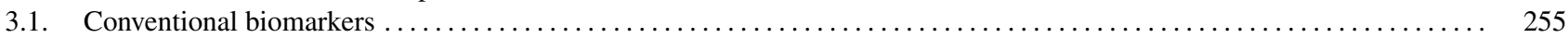

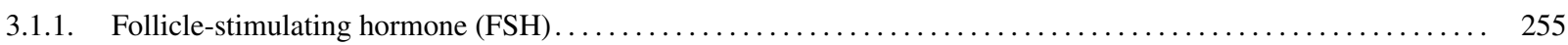

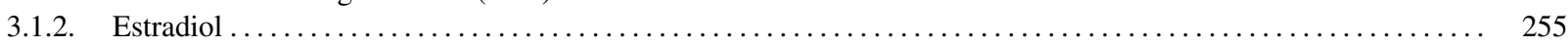

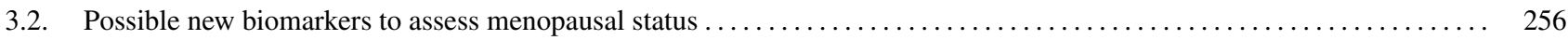

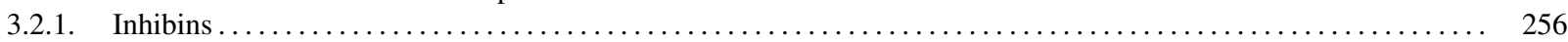

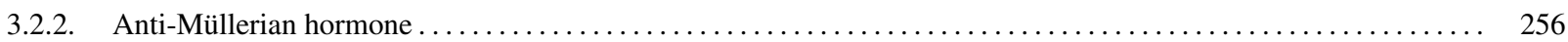

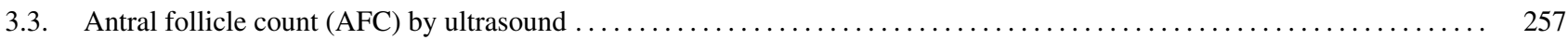

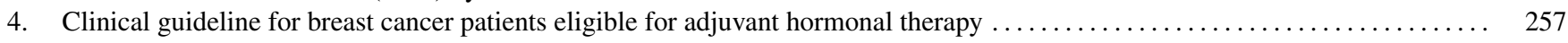

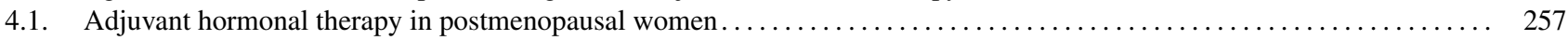

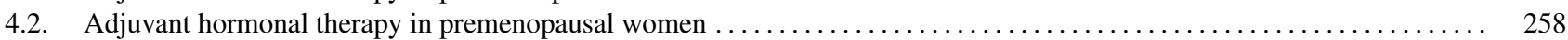

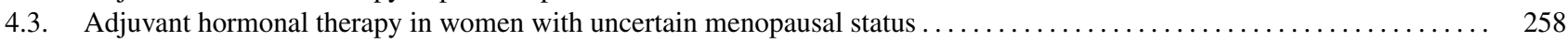

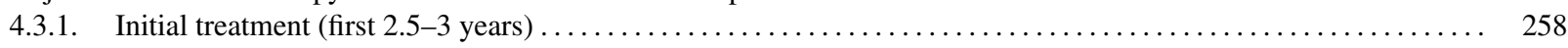

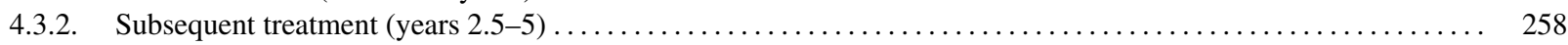

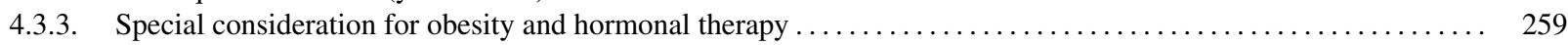

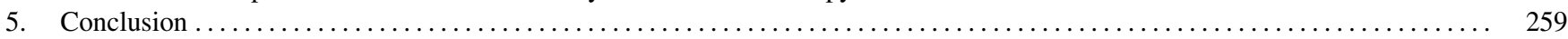

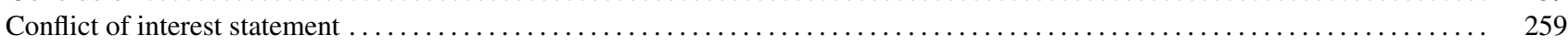

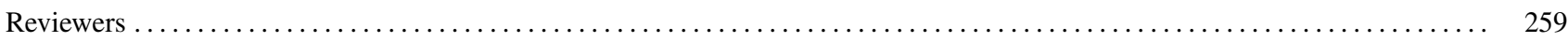

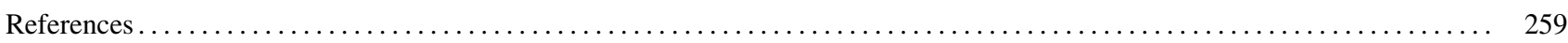

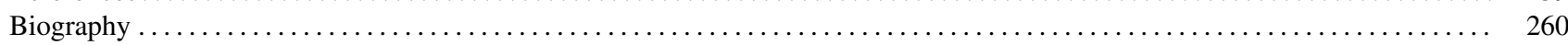

\footnotetext{
* Corresponding author at: Department of Medical Oncology (F02.126), University Medical Center Utrecht, Heidelberglaan 100, 3584 CX Utrecht, The Netherlands. Tel.: +31088 755 6265; fax: +31 0887553741 .

E-mail address: f.devos@umcutrecht.nl (F.Y.F.L. De Vos).
} 


\begin{abstract}
Breast cancer is the most common malignancy amongst women in the developed world. For patients with hormone-sensitive breast cancer eligible for adjuvant hormonal therapy, it is important to know if the ovaries are (still) functional or not. Indeed, the choice for a specific adjuvant hormonal treatment depends on the menopausal status of an individual woman. The currently available measures to determine the menopausal status are conflicting. Until better measures become available, we propose a practical guideline enabling an optimal choice of adjuvant hormonal therapy for women with a hormone receptor positive breast cancer taking into account uncertainties about their menopausal status.
\end{abstract}

(C) 2012 Elsevier Ireland Ltd. All rights reserved.

Keywords: Hormone-sensitive breast cancer; Menopausal status; Hormonal therapy

\section{Introduction}

Breast cancer is the most common malignancy among women, with a prevalence of almost 207,090 individuals in the United States in 2010. It is after colorectal cancer the second most common cause of cancer death in women, with an estimated 39,840 deaths in the same year [1]. Over the years, breast cancer mortality has declined partly due to the implementation of surveillance programs leading to early detection, and partly due to improved treatment modalities. One of these modalities consists of adjuvant endocrine therapy for hormone-sensitive breast cancer patients.

The choice for a specific hormonal treatment depends on the menopausal status of the patient. In postmenopausal patients, adjuvant hormonal treatment with an aromatase inhibitor (AI) given upfront or tamoxifen followed by an AI has proven to be superior to tamoxifen alone and therefore has become standard of care [2-5]. In contrast, in clearly premenopausal women adjuvant treatment with tamoxifen with or without ovarian suppression is recommended [6-9]. A randomized clinical trial comparing adjuvant ovarian suppression in combination with tamoxifen or anastrozole with or without zoledronic acid in premenopausal women with breast cancer who were not treated with chemotherapy has shown no superiority of an AI over tamoxifen in this artificially postmenopausal setting [10].

While tamoxifen can be safely given to premenopausal women, this is not the case for AIs. AIs interfere with the conversion of androgens into estrogens by blocking the enzyme aromatase resulting in low estradiol levels in truly postmenopausal women [11]. However, in the presence of (still) functional ovaries, low levels of estrogen will enhance pituitary FSH production indirectly stimulating the follicular production of aromatase and subsequently of estradiol. As a consequence, categorizing premenopausal women inaccurately as postmenopausal and treating them with AIs without co-treatment with a gonadotropin-releasing hormone (GnRH) agonist may be ineffective. Moreover, in case of chemotherapy induced amenorrhoea (CIA) AIs may promote recovery of ovarian function which could lead to therapeutic failure and even to unwanted pregnancy [12]. On the other hand, treatment with GnRH agonists may be accompanied with additional side effects and is costly [13].
In this review we will describe the physiology of natural reproductive aging, summarize the current methods to assess the menopausal status and propose a practical guideline for the optimal choice of adjuvant hormonal therapy for women with a hormone receptor positive breast cancer taking into account uncertainties about the exact menopausal status.

\section{Menopausal status}

\subsection{Physiology of natural reproductive aging}

The nomenclature for the different phases of reproductive aging has been inconsistent and confusing. In an attempt to uniform this nomenclature, the Stages of Reproductive Aging Workshop (STRAW) has developed a staging system providing useful clinical definitions of the reproductive stages including menopausal transition, perimenopause, final menstrual period, menopause, and post-menopause for healthy women aging to a natural menopause [14] (Fig. 1).

Folliculogenesis refers to the process during which primordial follicles are continuously recruited out of the primordial follicle pool. Recruited follicles develop via the primary, secondary and tertiary stages into antral (antrum containing) follicles [15] (Fig. 2). Up to a diameter of $10 \mathrm{~mm}$ follicle development is gonadotropin independent. After one follicle has been selected out of a small cohort of antral follicles measuring between 8 and $10 \mathrm{~mm}$, this follicle will become the dominant follicle. From this stage, final follicle development is dependent on follicle-stimulating hormone (FSH). The final maturation steps preceding ovulation are driven by FSH and luteinizing hormone (LH). Hence, final follicle development coincides with the onset of menses during which FSH levels are elevated. The 14-days follicular phase of the menstrual cycle represents the development of a late antral follicle into a pre-ovulatory follicle. At the end of this phase ovulation preludes the luteal phase of this menstrual cycle which takes another 14 days (Fig. 2). It is important to realize that the whole process lasts for approximately 360 days which is also roughly in accordance with the duration of the period between the final menstrual period and menopausal transition [16,17].

Ovaries of a newborn girl harbor about 1 million primordial follicles each. Due to a continuous process of atresia, 


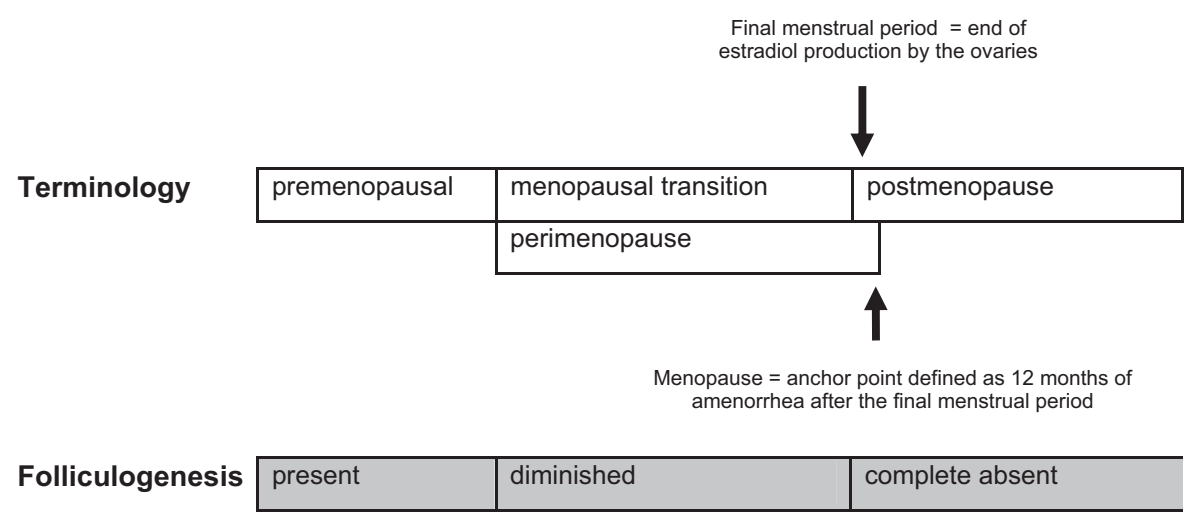

Fig. 1. Terminology of the different stages of the menopausal transition and the corresponding function of folliculogenesis.

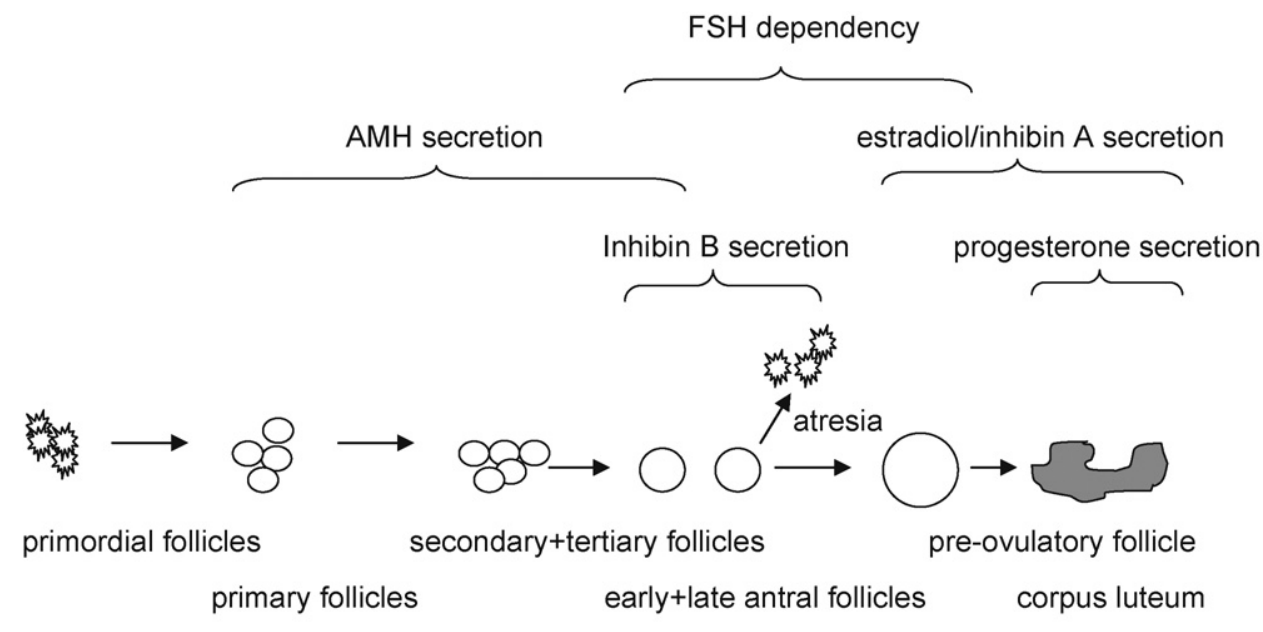

FOLLICULOGENESIS (360 days)

MENSTRUAL CYCLE (28 days)

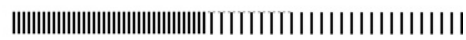

follicular phase luteal phase

Fig. 2. Folliculogenesis and menstrual cycle.

the majority of primordial follicles will be lost. At puberty, the total number per ovary has dropped to approximately 300,000-400,000. During a women's reproductive period only about 400 of these will eventually ovulate, while most follicles will disappear through atresia involving the process of apoptosis. At menopause, there is a nearly complete depletion of primordial follicles. With advanced age, decreasing number of oocytes and declining oocyte quality cause eventually the termination of estradiol production by the ovaries. The "final menstrual period" can only be defined in retrospect, i.e. after 12 months of amenorrhea. The average age of menopause in women of Caucasian/European origin is around 51 years of age, but can range from 40 up to 60 years [18].

\subsection{Defining menopausal status in breast cancer patients}

In general, three groups of women can safely be considered postmenopausal; all women older than the age of 60 , women who underwent a bilateral ovariectomy, and women younger than 60 years not using oral contraceptives or hormone replacement therapy (HRT) with an intact uterus and being amenorrheic for at least one year prior to the diagnosis of breast cancer. Women having regular menses/periods without using oral contraceptives or HRT can be classified as premenopausal. Strictly stated, remaining ovarian activity cannot be excluded for all other women and therefore the menopausal status is then uncertain. 


\subsubsection{Chemotherapy induced amenorrhea (CIA)}

Ascertainment of the menopausal status of breast cancer patients may be more complicated by the effect of anticancer therapy on functional ovarian reserve. Patients may develop CIA that could be transient. Bines et al. proposed to define CIA as secondary amenorrhea of $>6$ months in a patient being pre- or peri-menopausal before the start of chemotherapy [19]. However, an amenorrheic period after chemotherapy of even more than one year in a patient with an intact uterus might not exclude resumption of menses.

The effect of chemotherapy on the ovaries may consist of direct destruction of remnant functional follicles or induction of ovarian fibrosis with a total disappearance of functional follicles. On the other hand, chemotherapy could also lead to CIA by inducing primary or hypergonadotropic hypogonadism, as observed by low levels of estradiol and high levels of FSH and LH [19]. As long as treatment does not contain high dose of glucocorticoids, depressing ACTH production, the pituitary function seems not affected by chemotherapy.

The induction of CIA mainly depends on the age at administration and type of chemotherapy administrated. In general, patients above the age of 40 years have a far greater risk of developing CIA without recovery of ovarian function (77-100\%) compared with patients below the age of 40 years (0-40\%), irrespective whether alkylating- or anthracyclinecontaining chemotherapy has been administered [12,20,21]. The wide range in risk for permanent CIA in women older than 40 years mentioned in the literature may relate to variability of age groups, lack of consistent CIA definition and different hormonal treatment strategies used in the various (retrospective) trials. Although occurrence and duration of CIA was not influenced by tamoxifen, use of AIs induced a recovery of ovarian function in $27 \%$ compared to historical $0-11 \%$ of women older than 40 years $[12,20]$.

In view of the abovementioned, it may be clear that assessing the actual functionality of the remaining ovarian tissue in breast cancer patients eligible for adjuvant endocrine therapy is of utmost importance and challenging. The question is whether the blood levels of hormones and/or growth factors, secreted in the different stages of the folliculogenesis, could be of help to define the start of the menopause.

\section{Biomarkers for assessment of menopausal status}

\subsection{Conventional biomarkers}

\subsubsection{Follicle-stimulating hormone (FSH)}

Levels of FSH combined with levels of estradiol are often used to define the menopausal status of women before and after treatment with chemotherapy, although these determinations are far from ideal. FSH is produced by the anterior pituitary gland, by pulsatile releases of gonadotropin releasing hormone from the hypothalamus. In women, FSH stimulates the growth of the small antral follicles and finally causes selection of the follicle having the most FSH receptors which then will become the dominant pre-ovulatory follicle $[15,17]$ (Fig. 2). Follicular cells of developing pre-ovulatory follicles, also called granulosa cells, produce considerable amounts of estradiol which in turn exerts a negative feedback at the level of the pituitary hypothalamus and consequently FSH levels decrease. Elevated blood FSH levels reflect an age-dependent decrease in the follicle pool. Therefore, in time the number of follicles with properly functioning granulosa cells will decline and hence estradiol levels will decrease. Consequently FSH levels will rise. The so-called monotropic rise in FSH levels is the latest hormonal event preceding menopause. However, the elevation of FSH may already occur approximately 3-8 years before menopause, at least in the presence of anovulatory cycles $[22,23]$. As the FSH levels rise above $20 \mathrm{IU} / \mathrm{L}$ during the late perimenopausal phase, this is often used as the cut-off value to determine depletion of ovarian reserve [22-24]. However, treatment with tamoxifen in truly postmenopausal women may induce a decrease in FSH levels, even into the premenopausal range [25]. Conversely, chemotherapy-induced amenorrhea in premenopausal women may result in highly increased FSH levels which can be temporarily and as such is no guarantee for absence of folliculogenesis in the future [24]. Therefore, no absolute cut-off level of FSH can be given above which folliculogenesis does not occur anymore.

\subsubsection{Estradiol}

Estradiol is mainly secreted by the late antral follicle and the ensuing corpus luteum, controlled by FSH and LH [15]. Once the ovarian secretion of estradiol is absent, the blood levels of estradiol depend predominantly on the conversion of androstenedione-testosterone to estradiol by the aromatase enzyme in abdominal/peripheral fat and other tissues. It has been reported that estradiol levels lower than $130 \mathrm{pmol} / \mathrm{L}$ can be considered as postmenopausal levels, but values between 10 and $60 \mathrm{pmol} / \mathrm{L}$ have been reported as well $[12,22,23]$. Also, estradiol levels may be highly variable in postmenopausal women, as this is influenced by obesity/body mass index (BMI) and smoking habits [22,23]. Estradiol levels are higher in obese postmenopausal women due to an increased number of adipose cells and, therefore, relatively more aromatase activity, while nicotine and its metabolite cotinine are strong inhibitors of aromatase leading to lower levels of estradiol levels among smokers [26,27]. Finally, HRT might lower FSH and increase estradiol levels up to one year after cessation of therapy [28].

It is also important to mention that the accuracy of commercially available methods for determination of estradiol levels is largely variable. Most clinical assays in use are automated or kit-based immunoassays. Extraction and purification of estradiol from plasma are frequently skipped in the quantification process, as speed, ease of analysis and small sample volume were the preferred variables focusing on premenopausal estradiol levels of 100-1200 pmol/L. Competition between sex hormone binding globulin and the estradiol antibody used in these assays and the presence of 
water-soluble conjugated steroid concentrations lead to variable estradiol levels with an increasing risk for overestimating the estradiol concentration, especially in patients with low levels. Routine use of direct estrogen assays for control of AI treatment compliance is not recommended, as increased pre-aromatization steroid concentrations by inhibition of the enzyme may bias the results. Accuracy of measuring lower estradiol levels would be improved largely by immunoassays including an extraction step or tandem gas chromatography and mass spectrometry assays [29]. Further improvement of radioimmunoassays led to a lower detection level of estradiol $(0.67 \mathrm{pmol} / \mathrm{L})$, useful in postmenopausal women and women treated with AIs [30].

Thus, there is no clear cut-off level of estradiol which predicts the complete absence of folliculogenesis.

\subsection{Possible new biomarkers to assess menopausal status}

To assess menopausal status more accurately, it is rational to search for a hormone or a growth factor which is solely produced by the follicles during the early stages of the folliculogenesis (i.e. the primordial or primary follicles) and not only by follicles that have advanced to more mature stages. Theoretically, such a 'marker' will be undetectable if the primordial follicle pool is completely exhausted. This would tremendously facilitate the assessment of the menopausal status. Two possible candidates have been proposed: inhibin B and anti-Müllerian hormone (AMH).

\subsubsection{Inhibins}

Inhibins are members of the TGF- $\beta$ superfamily and composed of an $\alpha$ - and $\beta$-subunit forming inhibin $A$ or inhibin $B$, respectively [15] (Fig. 2). Like estradiol, inhibin A is secreted by pre-ovulatory follicles and the corpus luteum and, therefore, a less plausible predictive marker for ovarian reserve. Inhibin B is produced by early antral follicles. With a dwindling number of antral follicles in the late perimenopause, inhibin B levels begin to drop and, therefore, might be a good marker of ovarian reserve [28,31]. However, inhibin $\mathrm{B}$ levels are not constant during the menstrual cycle; i.e. they increase during the early follicular phase and decrease during the late follicular and early luteal phase following FSH feedback. Furthermore, serum inhibin B levels are already undetectable about 4 years prior to the final menstrual period $[32,33]$. Inhibin B has been investigated in a single study in 127 premenopausal, early breast cancer patients who were treated with chemotherapy [34]. Compared with age-matched controls, chemotherapy treated cancer subjects had significantly lower inhibin B levels $(p=0.001)$. Inhibin B was significantly associated with risk of CIA, even after controlling for FSH. These levels could possibly predict whether the ovarian reserve before the start of chemotherapy is sufficient to survive the chemotherapy induced loss of follicles. Further research in this field is warranted in order to obtain more information hereon.

\subsubsection{Anti-Müllerian hormone}

$\mathrm{AMH}$, also known as Müllarian inhibiting substance, is a member of the transforming growth factor-beta (TGF$\beta$ ) superfamily and is produced by the pool of primary, secondary and early antral follicles (Fig. 2). Levels of $\mathrm{AMH}$, undetectable during the first years after birth in girls, rise sharply during puberty when folliculogenesis starts and remains relatively unchanged throughout the menstrual cycles $[35,36]$. This is mainly due to the fact that, in contrast to estradiol, AMH does not exert any feedback on the hypothalamic-pituitary-ovarian axis [37,38].

The two main functions of AMH are: (1) inhibition of recruitment of primordial follicles; and (2) inhibition of FSH sensitivity of late antral follicles. As the pool of primordial follicles diminishes with aging, the follicle pool that secretes AMH decreases. As a consequence, lower levels of AMH will increase the rate of recruitment of primordial follicles, resulting in an acceleration of the depletion of the primordial follicle pool toward the end of the reproductive period. Therefore, reduced AMH levels could be an excellent early marker for ovarian reserve through the menopausal transition. In a recently published long-term follow-up study of normo-ovulatory women (age 21-46 yr), age-specific $\mathrm{AMH}$ levels indeed were highly predictive for timing the menopause [37].

Despite the potential of AMH as a marker of menopause several challenges remain. Although the currently available enzyme-immunometric assays are highly sensitive (detection level $0.05 \mathrm{ng} / \mathrm{ml}$ ), the lowest level of detection is still not an absolute cut-off level marking the menopause precisely [37]. Moreover, the effect of chemotherapy on and the interpretation of the AMH levels are still unclear. In the previously mentioned study by $\mathrm{Su}$ et al., breast cancer patients with stage I to III disease who were premenopausal at diagnosis and were enrolled post-chemotherapy, had significantly lower AMH levels compared to age-matched controls [34]. Low AMH levels of treated patients were significantly associated with the risk of CIA, also after controlling for FSH. Only two observational studies prospectively investigated AMH up to one year after the end of adjuvant chemotherapy in premenopausal breast cancer patients [39,40]. In the first study, fifty premenopausal women (age range 28-52 years) with early breast cancer were included [39]. Pretreatment, AMH concentrations were lower with increasing age even before changes in other hormone concentrations occurred. AMH concentration showed a rapid and marked fall during the chemotherapy period, reaching undetectable concentrations in many women $(p<0.0001)$. The second study described seventeen women undergoing chemotherapy (median age 30 years; range 19-35 years; eight diagnosed with breast cancer) [40]. AMH levels dropped from $2.7 \pm 1.0$ to $1.1 \pm 0.6$ and to $0.4 \pm 0.4 \mathrm{ng} / \mathrm{mL}$ immediately after one and two cycles of chemotherapy, respectively, suggesting that the gonadotoxic effect of chemotherapy involves primary, secondary and early antral follicles. However, the AMH levels in most patients subsequently rose approximately 8 months after the 


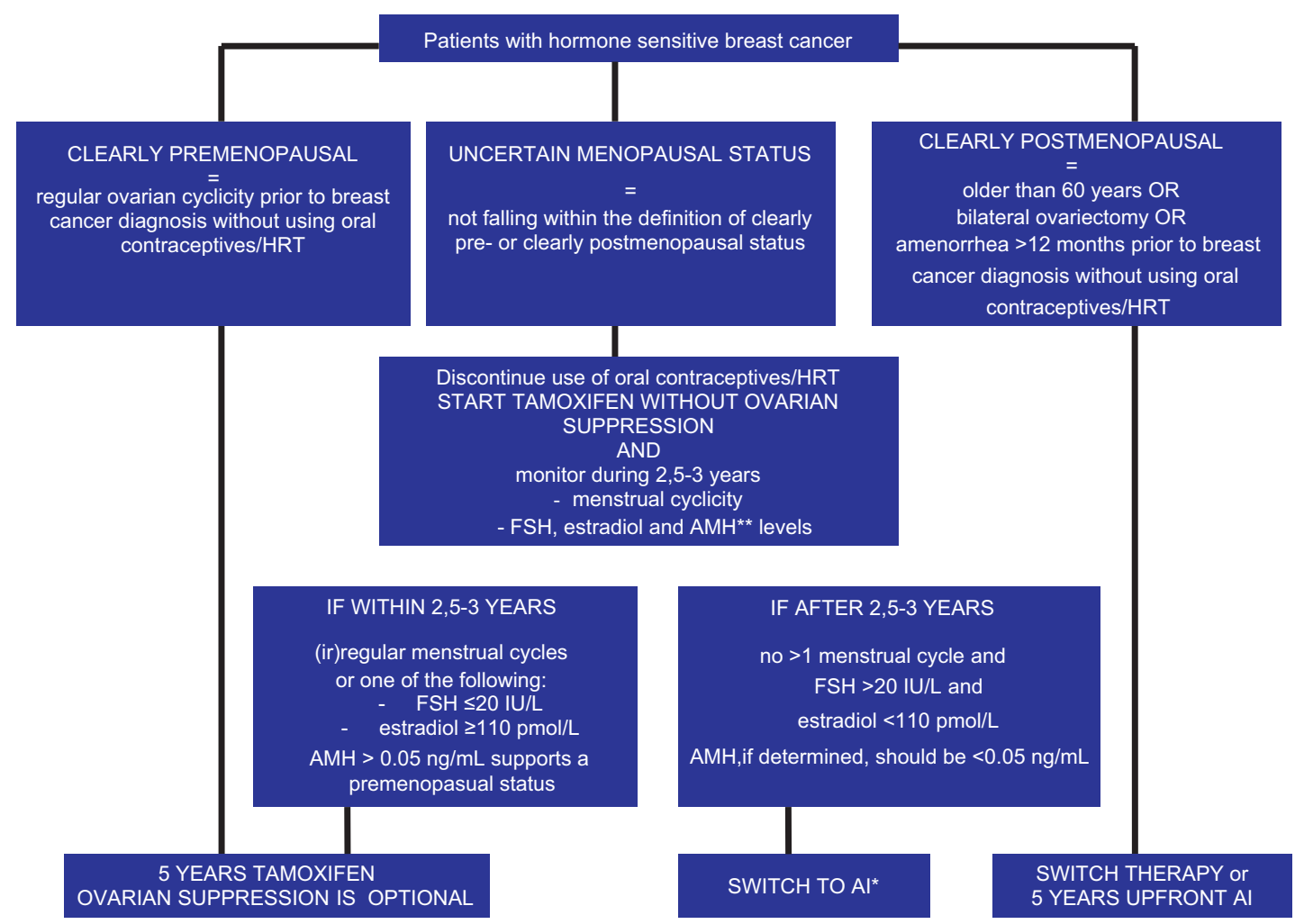

Fig. 3. Practical guideline for patients with hormone sensitive breast cancer with an indication for adjuvant hormonal therapy HRT, hormonal replacement therapy; AMH, anti-Müllerian hormone; FSH, follicle stimulating hormone; AI, aromatase inhibitor. * It remains still important for this group that women after starting AI should report reoccurrence of menses and/or declining of menopausal symptoms, with consequently measuring levels of FSH and estradiol e.g. every three months during one year. ** Only if local laboratory has the facility to measure AMH.

end of chemotherapy. Thus, a declined AMH level after start of chemotherapy is not always permanent and as such cannot predict whether folliculogenesis will or will not recover within the following year. Further research is needed to explore the exact predictive value of AMH levels in premenopausal early breast cancer patients before, after and up to at least one year after finishing adjuvant chemotherapy.

\subsection{Antral follicle count (AFC) by ultrasound}

Ovarian reserve is related to the number and quality of remaining primordial follicles. Unfortunately, these primordial follicles are too small to be visualized by ultrasound. In a study of 162 women (age 25-46 years) the number of antral follicles with a diameter between 2 and $10 \mathrm{~mm}$ was estimated by ultrasound in the early follicular phase and was suggested as a surrogate for ovarian reserve [41]. The number of small antral follicles in both ovaries as measured by ultrasound was related to reproductive age and could be a reflection of the size of the remaining primordial follicle pool. Similar to results found in healthy women, AFC is highly positively correlated with AMH levels in breast cancer survivors [42]. AFC was not an independent predictor of postmenopause in normo-ovulatory women [37]. Moreover, reproducibility of AFC is influenced by inter-observer and inter-cycle variability $[41,43]$. Finally, clinical use is hampered by the fact that AFC can only be assessed during the early follicular phase, while it remains unclear when AFC should be determined in women with oligo- or amenorrhea. Also, it is an elaborate and invasive (transvaginal) method to measure the ovarian reserve repeatedly during the first two years after finishing chemotherapy. Therefore, alternative methods are needed.

\section{Clinical guideline for breast cancer patients eligible for adjuvant hormonal therapy}

Given the difficulties as well as the relevance of determining the accurate menopausal status of breast cancer patients eligible for adjuvant hormonal therapy, we propose a practical guideline, based on the currently available evidence, without compromising the optimal hormonal treatment (Fig. 3).

\subsection{Adjuvant hormonal therapy in postmenopausal women}

In patients clearly classified as postmenopausal at the start of hormonal treatment (see Section 2.2), both switch therapy 
of 2-3 years of tamoxifen followed by 3-2 years of an AI or upfront AI for 5 years are adequate options [2-5].

\subsection{Adjuvant hormonal therapy in premenopausal women}

In clearly premenopausal women 5 years adjuvant treatment with tamoxifen with or without ovarian suppression is recommended [6].

\subsection{Adjuvant hormonal therapy in women with uncertain menopausal status}

\subsubsection{Initial treatment (first $2.5-3$ years)}

For patients in whom the menopausal status cannot yet be determined as truly postmenopausal at the start of hormonal treatment we propose to start tamoxifen without additional ovarian suppression. To date there is no convincing evidence for this particular group of mainly older pre- or perimenopausal women that adjuvant hormonal therapy with tamoxifen alone is inferior to tamoxifen with ovarian suppression, certainly after chemotherapy. A meta-analysis comparing tamoxifen alone versus tamoxifen combined with a GnRH agonist in premenopausal patients with hormone-receptor positive breast cancer showed no significant difference in disease-free and overall survival [44]. Furthermore, in a large retrospective study no significantly additional benefit from adding GnRH to tamoxifen compared to tamoxifen alone was observed, although in this study the tamoxifen dose was $40 \mathrm{mg}$ daily and the adjuvant hormonal therapy was ceased after 2 years [45]. At the Sankt Gallen Breast Cancer Conference 2011, only 37.5\% of the expert panel members favored combining ovarian suppression with tamoxifen to tamoxifen alone as adjuvant hormonal treatment in this group of premenopausal women [7]. This strategy is in line with the recently updated international guidelines of ASCO and NCCN recommending tamoxifen without ovarian suppression as a valid option in older premenopausal breast cancer patients [8,9]. Therefore, starting hormonal therapy with tamoxifen without ovarian suppression for $2-3$ years is a reasonable treatment option for patients with uncertain menopausal status post chemotherapy, and provides the opportunity to monitor the menopausal status within the following 2-3 years. Also, withholding the administration of GnRH agonist is less expensive, and more convenient for the patient. Of note, for patients younger than 40 years of age, a paucity of data is available supporting this general evidence of equivalence between tamoxifen and tamoxifen plus ovarian suppression. In fact, some data are in favor of the combined modality for this group [46-48]. Therefore, for patients younger than 40 years this guideline should be interpreted with caution.

In contrast herewith, Ortmann et al. make a plea for incorporating AI treatment in pre-, peri- or postmenopausal breast cancer patients as soon as possible [49]. E.g. in case of CIA and age $\geq 40$ years they suggest to start with upfront AI, if estradiol and FSH levels, assessed by reliable and valid measures, are in the postmenopausal range. Arguments in favor of our conservative approach may be found in the recently published article on the long-term efficacy data of the Austrian Breast and Colorectal Cancer Study Group trial12 (ABCSG-12) [50]. In this trial in premenopausal women with hormone-receptor positive early-stage (stage I-II) breast cancer receiving goserelin ( $3.6 \mathrm{mg}$ every 28 days) the efficacy and safety were compared of anastrozole ( $1 \mathrm{mg}$ per day) or tamoxifen ( $20 \mathrm{mg}$ per day) with or without zoledronic acid for 3 years. At a median follow-up of 62 months there was no difference in disease-free survival between patients on tamoxifen versus anastrozole, but overall survival was significantly worse for anastrozole compared to tamoxifen (46 versus 27 deaths; HR 1.75, 95\% CI 1.08-2.83; $p=0.02$ ). These data emphasize that tamoxifen is effective and safe, and underscore the use of tamoxifen in this patient group. Moreover, the initial treatment with AIs (with or without GnRH agonist) is not recommended for patients with an uncertain menopausal status $[6,12]$.

\subsubsection{Subsequent treatment (years 2.5-5)}

The decision whether or not to switch to an AI should be made after 2-3 years of adjuvant tamoxifen therapy, depending on whether or not a definite postmenopausal status has been attained. As discussed above, for the currently available biomarkers there is no specific cut off level to be used to determine a postmenopausal status with certainty. For current clinical practice and the decision to switch to an AI, we propose to use the re-occurrence of menses during the preceding two and a half year in combination with the results of serial measurements of FSH, estradiol and AMH levels (if the AMH measure is available). Since misdiagnosing patients as being postmenopausal should be avoided, it is sensible to consider a conservative definition of postmenopausal status. Therefore, we propose the following definition for postmenopause: no more than one menstrual period and FSH levels $>20 \mathrm{IU} / \mathrm{L}$ and estradiol levels in postmenopausal range according to the local laboratory (in general $<110 \mathrm{pmol} / \mathrm{L}$ ) over the $2-3$ years of tamoxifen treatment [22-24]. In hospitals where the determination of $\mathrm{AMH}$ is available, we would advise to incorporate an AMH level $<0.05 \mathrm{ng} / \mathrm{ml}$ as an additional condition for defining the postmenopause [40]. To increase accuracy, the determination of estradiol should preferably be performed by using immunoassays including an extraction step or tandem gas chromatography or by using mass spectrometry assays $[29,30]$. It should be noted that the predictive value of AMH in order to asses menopausal status is based on two trials only $[39,40]$.

Patients who meet the mentioned criteria can be considered "truly" postmenopausal and be switched from tamoxifen to an AI. After AI initiation, serial monitoring of FSH and estradiol is strongly recommended for at least one year after the start of AI administration. It still remains important to inform the women to report reoccurrence of menses and/or 
decline of menopausal symptoms, at which time levels of FSH and estradiol should be reassessed.

\subsubsection{Special consideration for obesity and hormonal therapy}

An increase in BMI leads to an increase in total-body aromatization and as a consequence, an increase in estradiol serum levels [51,52]. In other words BMI may serve as a useful surrogate parameter for total-body aromatization. As AIs inhibit the conversion of androgens to estrogens which occurs mainly in the peripheral apidose tissue, it is conceivable that the efficacy of AIs is influenced by the total-body aromatization and thus the patient's BMI $[26,53]$. Moreover, there could be an effect of BMI on the relative benefit of an AI versus tamoxifen.

Several retrospective studies have indeed suggested a nonsignificant trend in a greater relative efficacy of AIs versus tamoxifen in non-obese compared to obese post-menopausal patients [53,54]. Among premenopausal patients, the efficacy of anastrozole plus goserelin compared to tamoxifen plus goserelin was also non-significantly impaired (hazard rate of disease free survival $=1.49$ [range, $0.93-2.38$ ] $; p=0.088$ ) among those patients with a higher BMI $\left(25-29.9 \mathrm{~kg} / \mathrm{m}^{2}\right)$. The efficacy between the two treatment arms among normal weighted patients was not different [55]. Thus, the choice of adjuvant hormonal therapy in obese breast cancer patients is still a matter of debate. Prospective studies are urgently needed to clarify the precise efficacy of AIs in these patients.

\section{Conclusion}

For patients with hormone sensitive breast cancer, knowledge of the precise time point by which the ovarian reserve is depleted is of great importance for the decision regarding the optimal adjuvant hormonal treatment. Unfortunately, the currently available measures to determine the postmenopausal status are of limited reliability. Until better measures become available, we proposed a practical guideline based on the currently existing scientific evidence (Fig. 3). To validate the proposed guideline, we have set up a prospective follow-up trial to assess AMH as a predictive tool for recovery of the ovarian function following CIA.

\section{Conflict of interest statement}

The authors declare no conflict of interest.

\section{Reviewers}

Giuseppe Curigliano, $\mathrm{MD}, \mathrm{PhD}$, Istituto Europeo di Oncologia, Department of Medicine, Division of Medical Oncology, I-Milan, Italy.

Johann W.R. Nortier, MD, PhD, Leiden University Medical Center (LUMC), Department of Clinical Oncology, NL-2300 RC Leiden, Netherlands.

Tanja Cufer, MD, PhD, University Clinic Golnik, Medical Faculty, Golnik 4204, Slovenia.

\section{References}

[1] American Cancer Society. Cancer facts and figures 2010. Atlanta: American Cancer Society; 2010.

[2] Coombes RC, Kilburn LS. Time to TEAM: exemestane, or tamoxifen then exemestane? Lancet 2011;377:280-1.

[3] Cuzick J, Sestak I, Baum M, et al. Effect of anastrozole and tamoxifen as adjuvant treatment for early-stage breast cancer: 10-year analysis of the ATAC trial. Lancet Oncology 2010;11:1135-41.

[4] Dowsett M, Cuzick J, Ingle J, et al. Meta-analysis of breast cancer outcomes in adjuvant trials of aromatase inhibitors versus tamoxifen. Journal of Clinical Oncology 2010;28:509-18.

[5] van de Velde C, Rea D, Seynaeve C, et al. Adjuvant tamoxifen and exemestane in early breast cancer (TEAM): a randomised phase 3 trial. Lancet 2011;377:321-31.

[6] Early Breast Cancer Trialists' Collaborative Group (EBCTCG). Effects of chemotherapy and hormonal therapy for early breast cancer on recurrence and 15-year survival: an overview of the randomised trials. Lancet 2005;365:1687-717.

[7] Goldhirsch A, Wood WC, Coates AS, et al. Strategies for subtypes dealing with the diversity of breast cancer: highlights of the St Gallen International Expert Consensus on the primary therapy of early breast cancer 2011. Annals of Oncology 2011;22:1736-47.

[8] Burstein HJ, Prestrud AA, Seidenfeld J, et al. American Society of Clinical Oncology clinical practice guideline: update on adjuvant endocrine therapy for women with hormone receptor-positive breast cancer. Journal of Clinical Oncology 2010;28:3784-96.

[9] NCCN. NCCN Clinical Practice Guidelines in Oncology ${ }^{\mathrm{TM}}$ Breast Cancer - version 2.2011; 2011.

[10] Gnant M, Mlineritsch B, Schippinger W, et al. Endocrine therapy plus zoledronic acid in premenopausal breast cancer. New England Journal of Medicine 2009;360:679-91.

[11] Gruber CJ, Tschugguel W, Schneeberger C, Huber JC. Production and actions of estrogens. New England Journal of Medicine 2002;346:340-52.

[12] Smith IE, Dowsett M, Yap YS, et al. Adjuvant aromatase inhibitors for early breast cancer after chemotherapy-induced amenorrhoea: caution and suggested guidelines. Journal of Clinical Oncology 2006;24:2444-7.

[13] Robertson JF, Blamey RW. The use of gonadotrophin-releasing hormone $(\mathrm{GnRH})$ agonists in early and advanced breast cancer in pre- and perimenopausal women. European Journal of Cancer 2003;39: 861-9.

[14] Soules MR, Sherman S, Parrott E, et al. Executive summary: Stages of Reproductive Aging Workshop (STRAW) Park City, Utah, July, 2001. Menopause 2001;8:402-7.

[15] Knight PG, Glister C. TGF-beta superfamily members and ovarian follicle development. Reproduction 2006;132:191-206.

[16] Erickson GF, Shimasaki S. The physiology of folliculogenesis: the role of novel growth factors. Fertility and Sterility 2001;76:943-9.

[17] Fauser BC, Van Heusden AM. Manipulation of human ovarian function: physiological concepts and clinical consequences. Endocrine Reviews 1997;18:71-106.

[18] http://www.eurohealth.ie/newrep/trends.htm; 2011 [20-8-2011].

[19] Bines J, Oleske DM, Cobleigh MA. Ovarian function in premenopausal women treated with adjuvant chemotherapy for breast cancer. Journal of Clinical Oncology 1996;14:1718-29.

[20] Davis AL, Klitus M, Mintzer DM. Chemotherapy-induced amenorrhea from adjuvant breast cancer treatment: the effect of the addition of taxanes. Clinical Breast Cancer 2005;6:421-4.

[21] Walshe JM, Denduluri N, Swain SM. Amenorrhea in premenopausal women after adjuvant chemotherapy for breast cancer. Journal of Clinical Oncology 2006;24:5769-79.

[22] Burger HG, Hale GE, Dennerstein L, Robertson DM. Cycle and hormone changes during perimenopause: the key role of ovarian function. Menopause 2008;15:603-12.

[23] MacNaughton J, Banah M, McCloud P, Hee J, Burger H. Age related changes in follicle stimulating hormone, luteinizing hormone, 
oestradiol and immunoreactive inhibin in women of reproductive age. Clinical Endocrinology 1992;36:339-45.

[24] Han HS, Ro J, Lee KS, et al. Analysis of chemotherapy-induced amenorrhea rates by three different anthracycline and taxane containing regimens for early breast cancer. Breast Cancer Research and Treatment 2009;115:335-42.

[25] Rossi E, Morabito A, Di Rella F, et al. Endocrine effects of adjuvant letrozole compared with tamoxifen in hormone-responsive postmenopausal patients with early breast cancer: the HOBOE trial. Journal of Clinical Oncology 2009;27:3192-7.

[26] Cleland WH, Mendelson CR, Simpson ER. Effects of aging and obesity on aromatase activity of human adipose cells. Journal of Clinical Endocrinology and Metabolism 1985;60:174-7.

[27] Shulman A, Ellenbogen A, Maymon R, Bahary C. Smoking out the oestrogens. Human Reproduction 1990;5:231-3.

[28] Burger HG, Dudley EC, Hopper JL, et al. Prospectively measured levels of serum follicle-stimulating hormone, estradiol, and the dimeric inhibins during the menopausal transition in a population-based cohort of women. Journal of Clinical Endocrinology and Metabolism 1999;84:4025-30.

[29] Dowsett M, Folkerd E. Deficits in plasma oestradiol measurement in studies and management of breast cancer. Breast Cancer Research 2005;7:1-4.

[30] Geisler J, Eske D, Helle H, Duong NK, Lønning PE. An optimized, highly sensitive radioimmunoassay for the simultaneous measurement of estrone, estradiol and estrone sulfate in the ultra-low range in human plasma samples. Journal of Steroid Biochemistry and Molecular Biology 2008;109:90-5.

[31] Groome NP, Illingworth PJ, O'Brien M, et al. Measurement of dimeric inhibin B throughout the human menstrual cycle. Journal of Clinical Endocrinology and Metabolism 1996;81:1401-5.

[32] Sowers MR, Eyvazzadeh AD, McConnell D, et al. Anti-Mullerian hormone and inhibin $\mathrm{B}$ in the definition of ovarian aging and the menopause transition. Journal of Clinical Endocrinology and Metabolism 2008;93:3478-83.

[33] van Rooij IA, Broekmans FJ, Scheffer GJ, et al. Serum antimullerian hormone levels best reflect the reproductive decline with age in normal women with proven fertility: a longitudinal study. Fertility and Sterility 2005;83:979-87.

[34] Su HI, Sammel MD, Green J, et al. Antimullerian hormone and inhibin $\mathrm{B}$ are hormone measures of ovarian function in late reproductive-aged breast cancer survivors. Cancer 2010;116:592-9.

[35] La Marca A, Volpe A. Anti-müllerian hormone (AMH) in female reproduction: is measurement of circulating AMH a useful tool? Clinical Endocrinology 2006;64:603-10.

[36] Visser JA, Themmen AP. Anti-Mullerian hormone and folliculogenesis. Molecular and Cellular Endocrinology 2005;234:81-6.

[37] Broer SL, Eijkemans MJ, Scheffer GJ, et al. Anti-mullerian hormone predicts menopause: a long-term follow-up study in normoovulatory women. Journal of Clinical Endocrinology and Metabolism 2011;96:2532-9.

[38] Visser JA, de Jong FH, Laven JS, Themmen AP. Anti-Müllerian hormone: a new marker for ovarian function. Reproduction 2006;131: $1-9$.

[39] Anderson RA, Themmen AP, Al-Qahtani A, Groome NP, Cameron DA. The effects of chemotherapy and long-term gonadotrophin suppression on the ovarian reserve in premenopausal women with breast cancer. Human Reproduction 2006;21:2583-92.

[40] Rosendahl M, Andersen CY, La Cour Freiesleben N, Juul Am Løssl K, Andersen AN. Dynamics and mechanisms of chemotherapy-induced ovarian follicular depletion in women of fertile age. Fertility and Sterility 2010;94:156-66.

[41] Scheffer GJ, Broekmans FJ, Dorland M, Habbema JD, Looman CW, Te Velde ER. Antral follicle counts by transvaginal ultrasonography are related to age in women with proven natural fertility. Fertility and Sterility 1999;72:845-51.
[42] Partridge AH, Ruddy KJ, Gelber S, et al. Ovarian reserve in women who remain premenopausal after chemotherapy for early stage breast cancer. Fertility and Sterility 2010;94:638-44.

[43] Hansen KR, Morris JL, Thyer AC, Soules MR. Reproductive aging and variability in the ovarian antral follicle count: application in the clinical setting. Fertility and Sterility 2003;80:577-83.

[44] LHRH-agonists in Early Breast Cancer Overview group. Use of luteinising-hormone-releasing hormone agonists as adjuvant treatment in premenopausal patients with hormone-receptor-positive breast cancer: a meta-analysis of individual patient data from randomized adjuvant trials. Lancet 2007;369:1711-23.

[45] Sverrisdottir A, Johansson H, Johansson U, et al. Interaction between goserelin and tamoxifen in a prospective randomised clinical trial of adjuvant endocrine therapy in premenopausal breast cancer. Breast Cancer Research and Treatment 2011;128:755-63.

[46] The Adjuvant Breast Cancer Trials Collaborative Group. Ovarian ablation or suppression in premenopausal early breast cancer: results from the international adjuvant breast cancer ovarian ablation or suppression randomized trial. Journal of the National Cancer Institute 2007;99:516-25.

[47] Ahn SH, Son BH, Kim SW, et al. Poor outcome of hormone receptorpositive breast cancer at very young age is due to tamoxifen resistance: nationwide survival data in Korea: a report from the Korean Breast Cancer Society. Journal of Clinical Oncology 2007;25:2360-8.

[48] Parton M, Smith IE. Controversies in the management of patients with breast cancer: adjuvant endocrine therapy in premenopausal women. Journal of Clinical Oncology 2008;26:745-52.

[49] Ortmann O, Pagani O, Jones A, et al. Which factors should be taken into account in perimenopausal women with early breast cancer who may become eligible for an aromatase inhibitor? Recommendations of an expert panel. Cancer Treatment Reviews 2011;37:97-104.

[50] Gnant M, Mlineritsch B, Stoeger H, et al. Adjuvant endocrine therapy plus zoledronis acid in premenopausal women with early-stage breast cancer: 62-month follow-up from the ABCSG-12 randomised trial. Lancet Oncology 2011;12:631-41.

[51] Forney JP, Milewich L, Chen GT, et al. Aromatization of androstenedione to estrone by human adipose tissue in vitro. Correlation with adipose tissue mass, age, and endometrial neoplasia. Clinics in Endocrinology and Metabolism 1981;53:192-9.

[52] Longscope C, Baker R, Johnston Jr CC. Androgen and estrogen metabolism: relationship to obesity. Metabolism: Clinical and Experimental 1986;35:235-7.

[53] Wolters R, Schwentner L, Regierer A, Wischnewsky M, Kreienberg R, Wöckel A. Endocrine therapy in obese patients with primary breast cancer: another piece of evidence in an unfinished puzzle. Breast Cancer Research and Treatment 2012;131:925-31.

[54] Sestak I, Distler W, Forbes JF, Dowsett M, Howell A, Cuzick J. Effect of body mass index on recurrences in tamoxifen and anastrozole treated women: an exploratory analysis from the ATAC trial. Journal of Clinical Oncology 2010;28:3411-5.

[55] Pfeiler G, Königsberg R, Fesl C, et al. Impact of body mass index on the efficacy of endocrine therapy in premenopausal patients with breast cancer: an analysis of the prospective ABCSG-12 trial. Journal of Clinical Oncology 2011;29:3653-9.

\section{Biography}

Dr. Filip De Vos has been trained as a medical oncologist at the Erasmus MC in Rotterdam. Hereafter, he worked at the department of Medical Oncology of the Radboud University Nijmegen Medical Centre, and from November 2011 onwards, he works as a member of staff at the department of Medical Oncology of the University Medical Centre Utrecht. 